\title{
POLYNOMIAL RELATIONS AMONG CHARACTERS COMING FROM QUANTUM AFFINE ALGEBRAS
}

\author{
Michael Kleber
}

\begin{abstract}
The Jacobi-Trudi formula implies some interesting quadratic identities for characters of representations of $\mathfrak{g l}_{n}$. Earlier work of Kirillov and Reshetikhin proposed a generalization of these identities to the other classical Lie algebras, and conjectured that the characters of certain finite-dimensional representations of $U_{q}(\hat{\mathfrak{g}})$ satisfy it. Here we use a positivity argument to show that the generalized identities have only one solution.
\end{abstract}

\section{Introduction}

1.1. Motivation. The Jacobi-Trudi (or Giambelli) formula tells us that the Schur function of an arbitrary partition can be realized as the determinant of a matrix whose entries are homogeneous (or elementary) symmetric functions. In the language of representations of $\mathfrak{g l}_{n}$ indexed by Young diagrams, this says that the character of an arbitrary representation is a determinant of a matrix whose entries are characters for Young diagrams with a single row (or column).

Now look at representations corresponding to rectangular Young diagrams. The matrix coming from an $\ell \times(m+1)$ rectangle contains as minors the matrices corresponding to rectangles of sizes $(\ell-1) \times m,(\ell+1) \times m, \ell \times(m-1)$, and $\ell \times m$ in two different ways. The three-term Plücker relation then yields the following identity:

$$
Q_{m}(\ell)^{2}=Q_{m-1}(\ell) Q_{m+1}(\ell)+Q_{m}(\ell-1) Q_{m}(\ell+1),
$$

where $Q_{m}(\ell)$ is the character associated to the $\ell \times m$ rectangular Young diagram. This beautiful identity is not as well-known as it ought to be.

The representations whose Young diagrams are a single column are the fundamental representations of $\mathfrak{g l}_{n}$, and one might hope that a similar picture could be constructed starting with the fundamental representations of other Lie algebras. Unfortunately, one can easily check that determinants filled in with those fundamental characters do not give characters of actual representations. Variants on the Jacobi-Trudi identity for other groups do exist (see Appendix A.3 of $[\mathrm{FH}]$ ), but they do not behave well with respect to taking minors, and so do not yield analogs of equation (1).

We can hope for a more satisfactory generalization, though: perhaps we could start with some other representations of $\mathfrak{g}$, not necessarily irreducible, and build

Received November 9, 1998. R evised December 8, 1998. 
a set of representations made of their determinants which do satisfy relations like equation (1).

In 1987, Kirillov and Reshetikhin investigated certain representations of a recently-defined quantum deformation of the universal enveloping algebra of $\mathfrak{g}$. They conjectured that the analogs of fundamental representations for this algebra satisfied a generalization of equation (1). The representations were $\mathfrak{g}$ modules as well, so they formed a good generalization of the complete $\mathfrak{g l}_{n}$ picture.

In the present paper, we reverse this process. Beginning with the desire to generalize the $\mathfrak{g l}_{n}$ picture to types $B, C$ and $D$ and retain certain properties, we show that the Kirillov-Reshetikhin solution is in fact the only one, regardless of its interpretation in terms of quantum deformations.

1.2. Background. Let $\mathfrak{g}$ be a complex finite-dimensional simple Lie algebra, $\hat{\mathfrak{g}}$ its corresponding affine Lie algebra. Because of the inclusion of quantum enveloping algebras $U_{q}(\mathfrak{g}) \hookrightarrow U_{q}(\hat{\mathfrak{g}})$, any finite-dimensional representation of $U_{q}(\hat{\mathfrak{g}})$ is a direct sum of irreducible representations of $U_{q}(\mathfrak{g})$.

Here we are particularly interested in the representations of $U_{q}(\hat{\mathfrak{g}})$ whose highest weights are multiples of one of the fundamental weights $\omega_{1}, \ldots, \omega_{n}$ of $\mathfrak{g}$, $n=\operatorname{rank}(\mathfrak{g})$. Unfortunately, there is presently no character formula known for these modules in general. The decomposition into $U_{q}(\mathfrak{g})$-modules has been explored in $[\mathrm{KR}]$ and $[\mathrm{ChP}]$, and recently by the author in $[\mathrm{K}]$.

Let $Q_{m}(\ell)$ denote the character of a certain $U_{q}(\hat{\mathfrak{g}})$-module with highest weight $m \omega_{\ell}$, where $\ell=1, \ldots, n$ and $m$ is a nonnegative integer (see section 2.1 for precise definitions). Based on a conjectural formula for the values of the $Q_{m}(\ell)$, these characters appear to satisfy certain remarkable polynomial identities. When $\mathfrak{g}$ is simply-laced, the identities have the form

$$
Q_{m}(\ell)^{2}=Q_{m-1}(\ell) Q_{m+1}(\ell)+\prod_{\ell^{\prime} \sim \ell} Q_{m}\left(\ell^{\prime}\right)
$$

for each $\ell=1, \ldots, n$ and $m \geq 1$. The product is taken over all $\ell^{\prime}$ adjacent to $\ell$ in the Dynkin diagram of $\mathfrak{g}$. When $\mathfrak{g}=\mathfrak{g l}_{n}$ this is just equation (1); the relations in full generality are written down in Section 2.2. Using these relations, it is possible to write any character $Q_{m}(\ell)$ in terms of the characters $Q_{1}(\ell)$ of the fundamental representations of $U_{q}(\hat{\mathfrak{g}})$.

The main result of this paper is that, for classical Lie algebras $\mathfrak{g}$, these equations have only one solution where $Q_{m}(\ell)$ is the character of a $U_{q}(\mathfrak{g})$-module with highest weight $m \omega_{\ell}$. By this condition, we mean we require that $Q_{m}(\ell)$ is a positive integer linear combination of irreducible $U_{q}(\mathfrak{g})$-characters whose highest weights sit under $m \omega_{\ell}$. We use the polynomial relations to write some of the multiplicities with which the smaller representations appear in $Q_{m}(\ell)$ in terms of the multiplicities in the characters $Q_{1}(\ell)$. The resulting inequalities determine all of the multiplicities.

The author is grateful to N. Yu. Reshetikhin for suggestion of the problem and words of wisdom. The research was partly supported by an Alfred P. Sloan 
Doctoral Dissertation Fellowship, and partly conducted while visiting the Research Institute for Mathematical Sciences (RIMS), Kyoto, Japan, thanks to the generosity of T. Miwa.

\section{Polynomial relations}

2.1. Definitions. We let $\mathfrak{g}$ be a finite-dimensional complex simple Lie algebra of rank $n$, and $\hat{\mathfrak{g}}$ be its corresponding affine Lie algebra. We will concentrate on the classical families $A_{n}, B_{n}, C_{n}$ and $D_{n}$. Choose simple roots $\alpha_{1}, \ldots, \alpha_{n}$ and fundamental weights $\omega_{1}, \ldots, \omega_{n}$ of $\mathfrak{g}$.

We will study certain finite-dimensional representations $W_{m}(\ell)$ of $U_{q}(\hat{\mathfrak{g}})$, where $m=0,1,2, \ldots$ and $\ell=1, \ldots, n$. Since $U_{q}(\mathfrak{g})$ appears as a Hopf subalgebra of $U_{q}(\hat{\mathfrak{g}})$, we can talk about weights and characters of $U_{q}(\hat{\mathfrak{g}})$ modules by restricting our attention to the $U_{q}(\mathfrak{g})$ action. From this point of view, $W_{m}(\ell)$ has highest weight $m \omega_{\ell}$. The structure as a $U_{q}(\hat{\mathfrak{g}})$ module is determined by Drinfeld polynomials $P_{1}(z), \ldots, P_{n}(z)$ instead of weights; the polynomials for $W_{m}(\ell)$ are

$$
\begin{aligned}
& P_{\ell}(z)=\prod_{i=1}^{m}\left(z+\frac{\left(\alpha_{i}, \alpha_{i}\right)}{4}(m+1-2 i)\right), \\
& P_{k}(z)=1, \text { for } k \neq \ell
\end{aligned}
$$

Chari and Pressley have also developed the notion of a $U_{q}(\hat{\mathfrak{g}})$ module being a "minimal affinization" of an irreducible $U_{q}(\mathfrak{g})$ module; see [ChP] for details. In this language, our representation $W_{m}(\ell)$ is the unique minimal affinization of the irreducible representation of $U_{q}(\mathfrak{g})$ with highest weight $m \omega_{\ell}$.

Let $Q_{m}(\ell)$ denote the character of $W_{m}(\ell)$ viewed as a representation of $U_{q}(\mathfrak{g})$. If $m=0$ then $W_{m}(\ell)$ is the trivial representation and $Q_{m}(\ell)=1$. The objects $W_{1}(\ell)$ and $Q_{1}(\ell)$ are called the fundamental representations and characters.

Finally, let $V(\lambda)$ denote the character of the irreducible representation of $U_{q}(\mathfrak{g})$ with highest weight $\lambda$. We will write characters $Q_{m}(\ell)$ as sums $\sum m_{\lambda} V(\lambda)$. Determining the integers $m_{\lambda}$ is of interest in part because they are closely related to solutions of certain Bethe equations; this is the subject of $[\mathrm{KR}]$ and $[\mathrm{K}]$. We will refer to the coefficients $m_{\lambda}$ as the multiplicity of $V(\lambda)$ in the sum.

2.2. Relations. The characters $Q_{m}(\ell)$ when $\mathfrak{g}$ is of type $A_{n}$ satisfy equation (1), known to mathematical physicists as the "discrete Hirota relations." A conjectured generalization of these relations appears in $[\mathrm{KR}]$ for the classical Lie algebras, and appear as the "Q-system" in [KNS] for the exceptional cases as well. While we are only interested in the classical cases, we will give the relations in full generality.

For every positive integer $m$ and for $\ell=1, \ldots, n$,

$$
Q_{m}(\ell)^{2}=Q_{m+1}(\ell) Q_{m-1}(\ell)+\prod_{\ell^{\prime} \sim \ell} \mathcal{Q}\left(m, \ell, \ell^{\prime}\right) .
$$



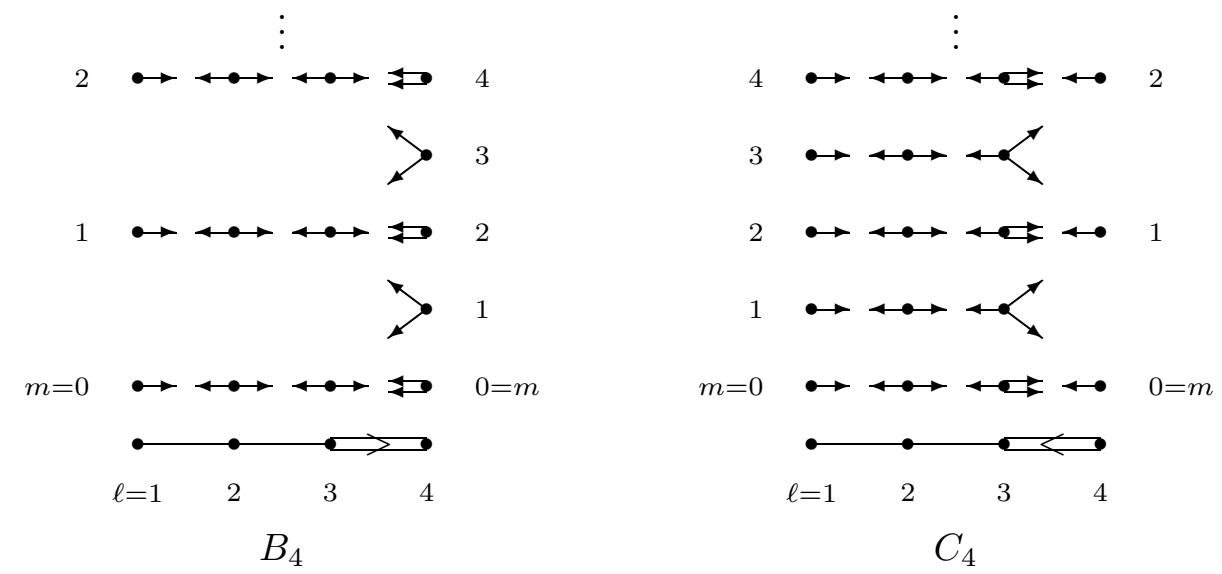

Figure 1. Product from the definition of $\mathcal{Q}$ for $B_{4}$ and $C_{4}$

The product is over all $\ell^{\prime}$ adjacent to $\ell$ in the Dynkin diagram of $\mathfrak{g}$, and the contribution $\mathcal{Q}\left(m, \ell, \ell^{\prime}\right)$ from $\ell^{\prime}$ is determined by the relative lengths of the roots $\alpha_{\ell}$ and $\alpha_{\ell^{\prime}}$, as follows:

$$
\mathcal{Q}\left(m, \ell, \ell^{\prime}\right)= \begin{cases}Q_{m}\left(\ell^{\prime}\right) & \text { if } \quad\left(\alpha_{\ell}, \alpha_{\ell}\right)=\left(\alpha_{\ell^{\prime}}, \alpha_{\ell^{\prime}}\right), \\ Q_{k m}\left(\ell^{\prime}\right) & \text { if } \quad\left(\alpha_{\ell}, \alpha_{\ell}\right)=k\left(\alpha_{\ell^{\prime}}, \alpha_{\ell^{\prime}}\right), \\ \prod_{i=0}^{k-1} Q_{\left\lfloor\frac{m+i}{k}\right\rfloor}\left(\ell^{\prime}\right) & \text { if } k\left(\alpha_{\ell}, \alpha_{\ell}\right)=\left(\alpha_{\ell^{\prime}}, \alpha_{\ell^{\prime}}\right),\end{cases}
$$

where $\lfloor x\rfloor$ is the greatest integer not exceeding $x$. We note that in the classical cases, the product differs from the simplified version in equation (2) only when:

$$
\begin{array}{rll}
\mathfrak{g}=\mathfrak{s o}(2 n+1), & \ell=n-1: & Q_{m}(n-2) Q_{2 m}(n) \\
& \ell=n: & Q_{\left\lfloor\frac{m}{2}\right\rfloor}(n-1) Q_{\left\lfloor\frac{m+1}{2}\right\rfloor}(n-1) \\
\mathfrak{g}=\mathfrak{s p}(2 n), & \ell=n-1: & Q_{m}(n-2) Q_{\left\lfloor\frac{m}{2}\right\rfloor}(n) Q_{\left\lfloor\frac{m+1}{2}\right\rfloor}(n) \\
& \ell=n: & Q_{2 m}(n-1)
\end{array}
$$

The structure of the product is easily represented graphically, with a vertex for each character $Q_{m}(\ell)$ and an arrow from $Q_{m}(\ell)$ pointing at each term of $\prod \mathcal{Q}\left(m, \ell, \ell^{\prime}\right)$; see Figure 1 for $\mathfrak{g}$ of type $B_{4}$ and $C_{4}$. The corresponding picture for $G_{2}$ is similarly pleasing.

Finally, we can solve equation (3) to get a recurrence relation:

$$
Q_{m}(\ell)=\frac{Q_{m-1}(\ell)^{2}-\prod \mathcal{Q}\left(m-1, \ell, \ell^{\prime}\right)}{Q_{m-2}(\ell)} .
$$

Note that the recurrence is well-founded: repeated use eventually writes everything in terms of the fundamental characters $Q_{1}(\ell)$. This is just the statement that iteration of "move down, then follow any arrow" in Figure 1 will eventually lead you from any point to one on the bottom row. In fact, $Q_{m}(\ell)$ is always 
a polynomial in the fundamental characters, though from looking at the recurrence it is only clear that it is a rational function. A Jacobi-Trudi style formula for writing the polynomial directly was given in $[\mathrm{KNH}]$.

The reason that characters of representations of quantum affine algebras are solutions to a discrete integrable system is still a bit of a mystery.

\section{Main Theorem}

3.1. Statement of the Main Theorem. The result of $[K R]$ was to conjecture a combinatorial formula for all the multiplicities $Z(m, \ell, \lambda)$ in the decomposition $Q_{m}(\ell)=\sum Z(m, \ell, \lambda) V(\lambda)$. We will refer to these proposed characters as "combinatorial characters" of the representations $W_{m}(\ell)$, although the conjecture that they are characters of some $U_{q}(\hat{\mathfrak{g}})$ module is unproven.

Theorem 1 (Kirillov-Reshetikhin). Let $\mathfrak{g}$ be of type $A, B, C$ or $D$. The combinatorial characters of $W_{m}(\ell)$ are the unique solution to equations (3) and (4) with the initial data

$$
\begin{aligned}
& A_{n}: Q_{1}(\ell)=V\left(\omega_{\ell}\right) \\
& 1 \leq \ell \leq n \\
& B_{n}: \quad Q_{1}(\ell)=V\left(\omega_{\ell}\right)+V\left(\omega_{\ell-2}\right)+V\left(\omega_{\ell-4}\right)+\cdots \quad 1 \leq \ell \leq n-1, \\
& Q_{1}(n)=V\left(\omega_{n}\right) \text {, } \\
& C_{n}: Q_{1}(\ell)=V\left(\omega_{\ell}\right) \quad 1 \leq \ell \leq n, \\
& D_{n}: \quad Q_{1}(\ell)=V\left(\omega_{\ell}\right)+V\left(\omega_{\ell-2}\right)+V\left(\omega_{\ell-4}\right)+\cdots \quad 1 \leq \ell \leq n-2, \\
& Q_{1}(\ell)=V\left(\omega_{\ell}\right) \quad \ell=n-1, n .
\end{aligned}
$$

The solutions $Q_{m}(\ell)$ to this recurrence are all characters of $U_{q}(\mathfrak{g})$, and the decomposition into irreducible representations is described combinatorially in terms of generalized "rigged configurations."

The explicit combinatorial formula for this solution, as given in the paper, is computationally intractable. An effective algorithm for computing this solution to the recurrence relations was given by the author in $[\mathrm{K}]$.

The main result of this paper is that the specification of initial data in Theorem 1 is unnecessary.

Theorem 2. Let $\mathfrak{g}$ be of type $A, B, C$ or $D$. The combinatorial characters of the representations $W_{m}(\ell)$ are the only solutions to equations (3) and (4) such that $Q_{m}(\ell)$ is a character of a representation of $U_{q}(\mathfrak{g})$ with highest weight $m \omega_{\ell}$, for every nonnegative integer $m$ and $1 \leq \ell \leq n$.

We need only prove that any choice of initial data other than that in Theorem 1 would result in some $Q_{m}(\ell)$ which is not a character of a representation of $U_{q}(\mathfrak{g})$. The values $Q_{m}(\ell)$ are always virtual $U_{q}(\mathfrak{g})$-characters, but in all other cases, some contain representations occurring with negative multiplicity. As an immediate consequence, we have: 
Corollary 3. If the characters $Q_{m}(\ell)$ of the representations $W_{m}(\ell)$ obey the recurrence relations in equations (3) and (4), then they must be given by the formula for combinatorial characters in $[\mathrm{KR}]$.

The technique of proof is as follows. The possible choices of initial data are limited by the requirement that $Q_{1}(\ell)$ be a representation with highest weight $\omega_{\ell}$. That is, $Q_{1}(\ell)$ must decompose into irreducible $U_{q}(\mathfrak{g})$-modules as

$$
Q_{1}(\ell)=V\left(\omega_{\ell}\right)+\sum_{\lambda \prec \omega_{\ell}} m_{\lambda} V(\lambda)
$$

Note that we require that $V\left(\omega_{\ell}\right)$ occur in $Q_{1}(\ell)$ exactly once. Furthermore, we require that for every other component $V(\lambda)$ that appears, $\lambda \prec \omega_{\ell}$, i.e., that $\omega_{\ell}-\lambda$ is a nonzero linear combination of simple roots with nonnegative integer coefficients.

We proceed with a case-by-case proof. For each series, we find explicit multiplicities of irreducible representations occurring in $Q_{m}(\ell)$ which would be negative for any choice of $Q_{1}(\ell)$ other than that of Theorem 1. The calculations for series $B, C$ and $D$ are found in sections $3.2,3.3$ and 3.4, respectively.

When $\mathfrak{g}$ is of type $A_{n}$, no computations are necessary, because every fundamental root is minuscule: there are no $\lambda \prec \omega_{\ell}$ to worry about, no other choices for initial data to rule out. In fact, $Q_{m}(\ell)$ is just $V\left(m \omega_{\ell}\right)$ for all $m$ and $\ell$, and moreover every $U_{q}(\mathfrak{g})$ module is also acted upon by $U_{q}(\hat{\mathfrak{g}})$, by means of the evaluation representation.

3.2. Series $B_{n}$. Let $\mathfrak{g}$ be of type $B_{n}$. Let $V_{i}$ stand for $V\left(\omega_{i}\right)$ for $1 \leq i \leq n-1$, and $V_{s p}$ for the character of the spin representation with highest weight $\omega_{n}$. For convenience, let $\omega_{0}=0$ and $V_{0}$ denote the character of the trivial representation. Finally, we denote by $V_{n}$ the character of the representation with highest weight $2 \omega_{n}$, which behaves like the fundamental representations.

There are no dominant weights $\lambda \prec \omega_{n}$, so $Q_{1}(n)=V_{s p}$. The only weights $\lambda \prec \omega_{a}$ are $0, \omega_{1}, \ldots, \omega_{a-1}$ for $1 \leq a \leq n-1$, so we write

$$
Q_{1}(a)=V_{a}+\sum_{b=0}^{a-1} M_{a, b} V_{b} .
$$

Our goal is to prove that the only possible values for the multiplicities are

$$
M_{a, b}= \begin{cases}1, & a-b \text { even } \\ 0, & a-b \text { odd }\end{cases}
$$

We will show these values are necessary inductively; the proof for each $M_{a, b}$ will assume the result for all $M_{c, d}$ with $\left\lceil\frac{c-d}{2}\right\rceil<\left\lceil\frac{a-b}{2}\right\rceil$ as well as those with $\left\lceil\frac{c-d}{2}\right\rceil=\left\lceil\frac{a-b}{2}\right\rceil$ and $c+d>a+b$. (Here $\lceil x\rceil$ is the least integer greater than or equal to $x$.) This amounts to working in the following order: 


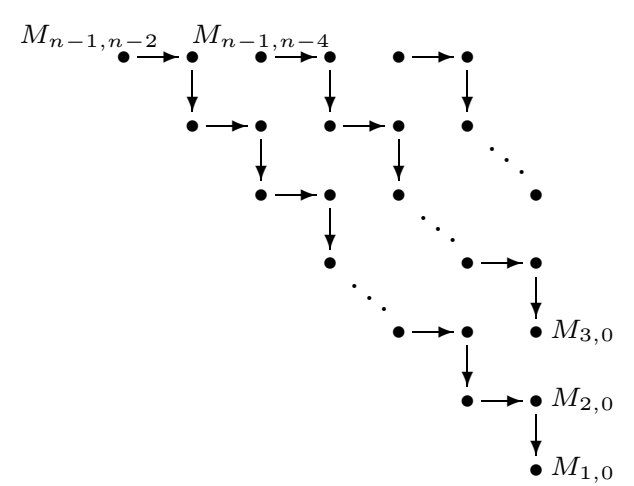

First follow the diagonal from $M_{n-1, n-2}$ to $M_{1,0}$, then the one from $M_{n-1, n-4}$ to $M_{3,0}$, etc., ending in the top right corner with $M_{n-1,0}$ or $M_{n-2,0}$, depending on the parity of $n$.

We show that equation (7) must hold for $M_{a, b}$, assuming it holds for all $M_{c, d}$ which appear earlier in this ordering, by the following calculations:

1. For $M_{n-1, b}$ where $n-1-b$ is odd, the multiplicity of $V\left(\omega_{b}+\omega_{n}\right)$ in $Q_{3}(n)$ is $1-2 M_{n-1, b}$,

2. For other $M_{a, b}$ where $a-b$ is odd, the multiplicity of $V\left(\omega_{a+2}+\omega_{b}\right)$ in $Q_{2}(a+1)$ is $-M_{a, b}$,

3. For $M_{a, b}$ where $a-b$ is even:

- The multiplicity of $V\left(\omega_{a}+\omega_{b}\right)$ in $Q_{2}(a)$ is $2 M_{a, b}-1$, and

- The multiplicity of $V\left(\omega_{a+2}+\omega_{b}\right)$ in $Q_{2}(a+1)$ is $1-M_{a, b}$.

Since all $M_{a, b}$ and all multiplicities are nonnegative integers, we must have $M_{a, b}=0$ to satisfy the first two cases and $M_{a, b}=1$ to satisfy the third.

The calculations to prove these claims depend on the ability to tensor together the $U_{q}(\mathfrak{g})$-modules whose characters form $Q_{m}(\ell)$. A complete algorithm for decomposing these tensors is given in terms of crystal bases in $[\mathrm{N}]$. For the current case, though, it happens that the only tensors we need to take are of fundamental representations. Simple explicit formulas for these decompositions had been given in $[\mathrm{KN}]$ before the advent of crystal base technology.

1. $M_{n-1, b}, n-1-b$ odd:

We want to find the multiplicity of $V\left(\omega_{b}+\omega_{n}\right)$ in $Q_{3}(n)$. Recursing through the polynomial relations, we find that

$$
Q_{3}(n)=Q_{1}(n)^{3}-2 Q_{1}(n) Q_{1}(n-1)=Q_{1}(n)\left[Q_{1}(n)^{2}-2 Q_{1}(n-1)\right] .
$$

Assuming equation (7) for $M_{n-1, b^{\prime}}$ for $b^{\prime}>b$ and recalling that $Q_{1}(n)^{2}=V_{s p}^{2}=$ $V_{n}+V_{n-1}+\cdots+V_{0}$, we need to compute the product

$$
V_{s p}\left[V_{n}-V_{n-1}+V_{n-2}-\cdots-V_{b+1}+\left(1-2 M_{n-1, b}\right) V_{b}-\cdots\right] .
$$

Since $V_{s p} V_{k}=\sum_{i=0}^{k} V\left(\omega_{i}+\omega_{n}\right)$, we find that the multiplicity of $V\left(\omega_{b}+\omega_{n}\right)$ in the product is the desired $1-2 M_{n-1, b}$.

2. $M_{a, b}, a-b$ odd, $a \leq n-2$ :

This calculation is typical of many of the ones that will follow, and will be 
written out in more detail. We want to know the multiplicity of $V\left(\omega_{a+2}+\omega_{b}\right)$ in $Q_{2}(a+1)$. When $a \leq n-3$, we have

$$
Q_{2}(a+1)=Q_{1}(a+1)^{2}-Q_{1}(a+2) Q_{1}(a) .
$$

Assuming inductively that equation (7) holds for all $M_{c, d}$ that precede $M_{a, b}$ in our ordering, we have

$$
\begin{aligned}
Q_{1}(a+1) & =V_{a+1}+V_{a-1}+\cdots+V_{b}+M_{a+1, b-1} V_{b-1}+\cdots \\
Q_{1}(a+2) & =V_{a+2}+V_{a}+\cdots+V_{b+1}+M_{a+2, b} V_{b}+\cdots, \\
Q_{1}(a) & =V_{a}+V_{a-2}+\cdots+V_{b+1}+M_{a, b} V_{b}+\cdots
\end{aligned}
$$

To compute $Q_{1}(a+1)^{2}-Q_{1}(a+2) Q_{1}(a)$, we note that the $V_{s} V_{t}$ term in $Q_{1}(a+1)^{2}$ and the $V_{s+1} V_{t-1}$ term in $Q_{1}(a+2) Q_{1}(a)$ are almost identical: when $s>t$, for example, the difference is just $\sum_{i=0}^{t} V\left(\omega_{i}+\omega_{s-t-2+i}\right)$. In our case, the only $V\left(\omega_{a+2}+\omega_{b}\right)$ term that does not cancel out is the one contributed by $M_{a, b} V_{a+2} V_{b}$, and the multiplicity of $V\left(\omega_{a+2}+\omega_{b}\right)$ is $-M_{a, b}$.

When $a=n-2$ the polynomial relations instead look like

$$
Q_{2}(n-1)=Q_{1}(n-1)^{2}-Q_{1}(n)^{2} Q_{1}(n-2)+Q_{1}(n-1) Q_{1}(n-2) .
$$

The $V_{n}+V_{n-2}+\cdots$ terms of $Q_{1}(n)^{2}$ behave just like the $Q_{1}(a+2)$ term above. The extra terms from $Q_{1}(n-2)\left[Q_{1}(n-1)-V_{n-1}-V_{n-3}-\cdots\right]$ make no net contribution, as can be seen by checking highest weights.

3. $M_{a, b}, a-b$ even:

Calculating the multiplicity of $V\left(\omega_{a+2}+\omega_{b}\right)$ in $Q_{2}(a+1)$ is similar to the above; the trick of canceling $V_{s} V_{t}$ with $V_{s+1} V_{t-1}$ works again. The only terms remaining are +1 from $V_{a+1} V_{b+1}$ and the same $-M_{a, b}$ from $V_{a+2} M_{a, b} V_{b}$ as above, so the multiplicity is $1-M_{a, b}$.

Likewise, calculating the multiplicity of $V\left(\omega_{a}+\omega_{b}\right)$ in $Q_{2}(a)$ we find two contributions of $M_{a, b}$ from $M_{a, b} V_{a} V_{b}$ (in either order) in $Q_{2}(a)^{2}$, and a contribution of 1 from $V_{a+1} V_{b+1}$ in $Q_{1}(a+1) Q_{1}(a-1)$, so the multiplicity is $2 M_{a, b}-1$.

3.3. Series $C_{n}$. Let $\mathfrak{g}$ be of type $C_{n}$. We let $V_{i}$ stand for $V\left(\omega_{i}\right)$ for $1 \leq i \leq n$. The only dominant weights $\lambda \prec \omega_{a}$ for $1 \leq a \leq n$ are $\lambda=\omega_{b}$ for $0 \leq b<a$ and $a-b$ even, where $\omega_{0}=0$. (If $a-b$ is odd, then $\omega_{a}$ and $\omega_{b}$ lie in different translates of the root lattice, so are incomparable.) So we write

$$
Q_{1}(a)=V_{a}+\sum_{i=0}^{\lfloor a / 2\rfloor} M_{a, a-2 i} V_{a-2 i} .
$$

We will prove that in fact $M_{a, b}=0$ for all $a$ and $b$.

Again we choose a convenient order to investigate the multiplicities: first look at $M_{a, a-2}$ for $a=n, n-1, \ldots, 2$, and then all $M_{a, b}$ with $a-b=4,6,8, \ldots$ This time the multiplicities acting as witnesses are: 
1. For $M_{a, a-2}$, the multiplicity of $V\left(\omega_{a-1}+2 \omega_{a-2}\right)$ in $Q_{3}(a-1)$ is $1-$ $2 M_{a, a-2}$,

2. For $M_{a, b}$ for $a-b \geq 4$, the multiplicity of $V\left(\omega_{a-2}+\omega_{b}\right)$ in $Q_{2}(a-1)$ is $-M_{a, b}$.

Performing these computations requires the ability to tensor more general representations of $\mathfrak{g}$ than were needed in the $B_{n}$ case. For this we use the generalization of the Littlewood-Richardson rule to all classical Lie algebras given in $[\mathrm{N}]$, which we summarize briefly in an Appendix.

1. $M_{a, a-2}$ :

We want to calculate the multiplicity of $V\left(\omega_{a-1}+2 \omega_{a-2}\right)$ in $Q_{3}(a-1)$. First we write $Q_{3}(a-1)$ as a sum of terms of the form $Q_{1}(x) Q_{1}(y) Q_{1}(z)$, which we denote as $(x ; y ; z)$ for brevity. When $2 \leq a-1 \leq n-2$, we have

$$
\begin{aligned}
& Q_{3}(a-1)=(a-1 ; a-1 ; a-1)-2(a ; a-1 ; a-2) \\
& -(a+1 ; a-1 ; a-3)+(a ; a ; a-3)+(a+1 ; a-2 ; a-2) .
\end{aligned}
$$

When $a-1$ is one of 1,2 or $n-1$, the above decomposition still holds, if we set $Q_{1}(0)=1$ and $Q_{1}(-1)=Q_{1}(n+1)=0$. We want to find the multiplicity of $V\left(\omega_{a-1}+2 \omega_{a-2}\right)$ in each of these terms.

First, $V\left(\omega_{a-1}+2 \omega_{a-2}\right)$ occurs with multiplicity 3 in the $V_{a-1}^{3}$ component of $Q_{1}(a-1)^{3}$. We calculate this number using the crystal basis technique for tensoring representations. Beginning with the Young diagram of $V_{a-1}$, we must choose a tableau $1,2, \ldots, a-2, p$ from the second tensor factor, where $p$ must be be one of $a-1, a$, or $\overline{a-1}$. Then the choice of tableau from the third tensor component must be the same but replacing $p$ with $\bar{p}$.

Similarly, the $V_{a} V_{a-1} V_{a-2}$ component of the $(a ; a-1 ; a-2)$ term produces $V\left(\omega_{a-1}+2 \omega_{a-2}\right)$ with multiplicity 1 , corresponding to the choice of the tableau $1,2, \ldots, a-2, \bar{a}$ from the crystal of $V_{a-1}$. We see that the remaining three terms cannot contribute by looking at tableaux in the same way.

Second, $V\left(\omega_{a-1}+2 \omega_{a-2}\right)$ occurs in the $M_{a, a-2} V_{a-2} V_{a-1} V_{a-2}$ piece of ( $a ; a-$ $1 ; a-2)$ and the $M_{a+1, a-1} V_{a-1} V_{a-2} V_{a-2}$ piece of $(a+1 ; a-2 ; a-2)$ as the highest weight component. Our inductive hypothesis, however, assumes that $M_{a+1, a-1}=0$, and we start the induction with $a=n$, where the $(a+1 ; a-2 ; a-2)$ term vanishes entirely.

Totaling these results, we find that the net multiplicity is $1-2 M_{a, a-2}$, and conclude that $M_{a, a-2}=0$.

2. $M_{a, b}$ for $a-b \geq 4$ :

We want to calculate the multiplicity of $V\left(\omega_{a-2}+\omega_{b}\right)$ in $Q_{2}(a-1)$. For any $2 \leq a-1 \leq n-1$, we have

$$
\begin{aligned}
Q_{2}(a-1) & =Q_{1}(a-1)^{2}-Q_{1}(a) Q_{1}(a-2) \\
& =\left(V_{a-1}+\cdots\right)\left(V_{a-1}+\cdots\right)-\left(V_{a}+M_{a, b} V_{b}+\cdots\right)\left(V_{a-2}+\cdots\right),
\end{aligned}
$$


where every omitted term is either already known to be 0 by induction, or else has highest weight less than $\omega_{b}$, so cannot contribute. As in the $B_{n}$ case, the $V_{a-1}^{2}$ and $V_{a} V_{a-2}$ terms nearly cancel one another's contributions: their difference is just $\sum_{k=0}^{a-1} V\left(2 \omega_{k}\right)$. Since $V\left(\omega_{a-2}+\omega_{b}\right)$ occurs in $V_{a-2} V_{b}$ with multiplicity 1 , the net multiplicity in $Q_{2}(a-1)$ is $-M_{a, b}$, and we conclude that $M_{a, b}=0$.

3.4. Series $D_{n}$. Let $\mathfrak{g}$ be of type $D_{n}$. This time we let $V_{i}$ stand for $V\left(\omega_{i}\right)$ for $1 \leq i \leq n-2$, and use $V_{n-1}$ for the character of the representation with highest weight $\omega_{n-1}+\omega_{n}$. We will not need to explicitly use the characters of the two spin representations individually, only their product, $V_{n-1}+V_{n-3}+\cdots$.

There are no dominant weights under $\omega_{n-1}$ or $\omega_{n}$, and so no work to do on $Q_{1}(n-1)$ or $Q_{1}(n)$. For $1 \leq a \leq n-2$, the only dominant weights $\lambda \prec \omega_{a}$ are $\lambda=\omega_{b}$ for $0 \leq b<a$ and $a-b$ even; again $\omega_{0}=0$. (If $a-b$ is odd, then $\omega_{a}$ and $\omega_{b}$ lie in different translates of the root lattice, so are incomparable.) So we write

$$
Q_{1}(a)=V_{a}+\sum_{i=0}^{\lfloor a / 2\rfloor} M_{a, a-2 i} V_{a-2 i} .
$$

We will show that in fact $M_{a, b}=1$ for all $a$ and $b$.

Again the proof is by induction; to show $M_{a, b}=1$ we will assume $M_{c, d}=1$ as long as either $c-d<a-b$ or $c-d=a-b$ and $c>a$. (This is the same ordering used for the $B_{n}$ series after dropping the $M_{a, b}$ with $a-b$ odd.) Our witnesses this time are:

- The multiplicity of $V\left(2 \omega_{b}\right)$ in $Q_{2}(a-1)$ is $1-M_{a, b}$, and

- The multiplicity of $V\left(\omega_{a}+\omega_{b}\right)$ in $Q_{2}(a)$ is $2 M_{a, b}-1$.

We must therefore conclude that $M_{a, b}=1$. Since we only need to tensor fundamental representations together, the explicit formulas given in $[\mathrm{KN}]$ are enough to carry out these calculations.

For any $\ell \leq n-3$, the polynomial relations give us

$$
Q_{2}(\ell)=Q_{1}(\ell)^{2}-Q_{1}(\ell+1) Q_{1}(\ell-1) .
$$

The multiplicity of $V\left(2 \omega_{b}\right)$ in $Q_{2}(a-1)$ is easily calculated directly, since $V\left(2 \omega_{b}\right)$ appears in $V_{r} V_{s}$ if and only if $r=s \geq b$, and then it appears with multiplicity one. The $Q_{1}(a-1)$ term therefore contains $V\left(2 \omega_{b}\right)$ exactly $(a-b) / 2$ times, while the $Q_{1}(a) Q_{1}(a-2)$ term subtracts off $M_{a, b}-1+(a-b) / 2$ of them. Thus the net multiplicity is $1-M_{a, b}$.

To calculate the multiplicity of $V\left(\omega_{a}+\omega_{b}\right)$ in $Q_{2}(a)$ for $a \leq n-3$, we once again use the trick of canceling the contribution from the $V_{s} V_{t}$ term of $Q_{1}(a)^{2}$ with the $V_{s+1} V_{t-1}$ term of $Q_{1}(a+1) Q_{1}(a-1)$. The cancellation requires more attention this time, since $V\left(\omega_{a}+\omega_{b}\right)$ occurs with multiplicity two in $V_{s} V_{t}$ when $a-b \geq 2 n-r-s$. In the end, the only terms that do not cancel are the contributions of $M_{a, b}$ from $V_{a} V_{b}$ and $V_{b} V_{a}$ in $Q_{1}(a)^{2}$ and of -1 from $V_{b+1} V_{a-1}$ in $Q_{1}(a+1) Q_{1}(a-1)$. Thus the net multiplicity is $2 M_{a, b}-1$. 
Finally, if $a=n-2$ the polynomial relations change to

$$
Q_{2}(n-2)=Q_{1}(n-2)^{2}-Q_{1}(n-1) Q_{1}(n) Q_{1}(n-3) .
$$

This change does not require any new work, though: $Q_{1}(n-1) Q_{1}(n)$ is just the product of the two spin representations, which decomposes as $V_{n-1}+V_{n-3}+\cdots$. Since this is exactly what we wanted $Q_{1}(\ell+1)$ to look like in the above argument, the preceding calculation still holds.

\section{Appendix: Littlewood-Richardson Rule for $C_{n}$}

This is a brief summary of a generalization of the Littlewood-Richardson rule to Lie algebras of type $C_{n}$, as given in $[\mathrm{N}]$. For our purposes, we only need the ability to tensor an arbitrary representation with one of the fundamental representations with highest weights $\omega_{1}, \ldots, \omega_{n}$.

The representation with highest weight $\sum_{k=1}^{n} a_{k} \omega_{k}$ is represented by a Young diagram $Y$ with $a_{k}$ columns of height $k$. For a fundamental representation $V_{k}$, we create Young tableaux from our column of height $k$ by filling in the boxes with $k$ distinct symbols $i_{1}, \ldots, i_{k}$ chosen in order from the sequence $1,2, \ldots, n, \bar{n}, \ldots, \overline{2}, \overline{1}$ in all possible ways, as long as if $i_{a}=p$ and $i_{b}=\bar{p}$ then $a+(k-b+1) \leq p$. These tableaux label the vertices of the crystal graph of the representation $V_{k}$.

Given a Young diagram $Y$, the symbols $1,2, \ldots, n$ act on it by adding one box to the first, second, $\ldots, n$th row, and the symbols $\overline{1}, \overline{2}, \ldots, \bar{n}$ act by removing one, provided the addition or removal results in a diagram whose rows are still nonincreasing in length. The result of the action of the symbol $i_{a}$ on $Y$ is denoted $Y \leftarrow i_{a}$.

Then the tensor product $V \otimes V_{k}$, where $V$ has Young diagram $Y$, decomposes as the sum of all representations with diagrams $\left(\left(\left(Y \leftarrow i_{1}\right) \leftarrow i_{2}\right) \cdots \leftarrow i_{k}\right)$, where $i_{1}, \ldots, i_{k}$ range over all tableaux of $V_{k}$ such that each of the actions still result in a diagram whose rows are still nonincreasing in length.

\section{References}

[ChP] V. Chari and A. Pressley. Quantum affine algebras and their representations. Representations of groups (Banff, AB, 1994), 59-78, CMS Conf. Proc. 16, Amer. Math. Soc., Providence, RI, 1995.

[FH] W. Fulton and J. Harris. Representation Theory. A first course. Graduate Texts in Mathematics, 129. Readings in Mathematics. Springer-Verlag, New York, 1991.

[KN] G. Kempf and L. Ness. Tensor products of fundamental representations. Canad. J. Math. 40 (1988), 633-648.

[KR] A.N. Kirillov and N.Yu. Reshetikhin. Representations of Yangians and multiplicities of occurrence of the irreducible components of the tensor product of representations of simple Lie algebras. (Russian) Zap. Nauchn. Sem. Leningrad. Otdel. Mat. Inst. Steklov. (LOMI) 160 (1987), transl. in J. Soviet Math. 52 (1990), 3156-3164.

[K] M. Kleber. Combinatorial structure of finite dimensional representations of Yangians: the simply-laced case. Internat. Math. Res. Notices (1997), 187-201.

$[\mathrm{KNH}]$ A. Kuniba, S. Nakamura, and R. Hirota. Pfaffian and determinant solutions to a discretized Toda equation for $B_{r}, C_{r}$ and $D_{r}$. J. Phys. A. 29 (1996), 1759-1766. 
[KNS] A. Kuniba, T. Nakanashi, and J. Suzuki. Functional relations in solvable lattice models: I. functional relations and representation theory. Internat. J. Modern Phys. A 9 (1994), 5215-5266.

[N] T. Nakashima. Crystal Base and a Generalization of the Littlewood-Richardson Rule for the Classical Lie Algebras. Comm. Math. Phys. 154 (1993), 215-243.

Massachusetts Institute of Technology, Department of Mathematics, 77 Massachusetts Ave, Cambridge, MA 02139.

E-mail address: kleber@math.mit.edu 